ИЗВЕСТИЯ АКАДЕМИИ НАУК ЭСТОНСКОН ССР. ТОМ ХV

СЕРИЯ ФИЗИКО-МАТЕМАТИЧЕСКИХ И ТЕХНИЧЕСКИХ НАУК. 1966, №

\title{
АЛГОРИТМ МЕТОДА ТРЕХМЕРНЫХ СЕТОК ДЛЯ АНАЛИЗА ДИНАМИЧЕСКИХ ПЕРЕХОДНЫХ ПРОЦЕССОВ ОСЕСИММЕТРИЧНОЙ ДЕФОРМАЦИИ ЦИЛИНДРИЧЕСКОЙ ОБОЛОЧКИ
}

Динамические переходные процессы деформации оболочек, вызванные внезапно приложенной или кратковременно действующей нагрузкой, теоретически мало исследованы, причем имеющиеся результаты [1] получены на основе приближенных теорий, обоснованность которых в начале движения вызывает определенные сомнения. Поэтому представляет интерес исследовать начало движения на основе теорин упругости. Если переходный процесс зависит от двух координат, изменяющихся в пределах прямоугольной области (плоская деформация плиты, осесимметричные задачи цилиндрической и конической оболочки), то решение уравнений теории упругости может быть построено методом трехмерных прямоугольных сеток.

Для переходных процессов типа плоской деформации плиты метод трехмерных сеток был предложен и использован в работе [2], причем программу для расчета на ЭЦВМ разработала А. Мянниль [3]. Ниже описываются алгоритм и программа метода трехмерных сеток для переходного волнового процесса осесимметрнчной деформацин цнлиндрических оболочек.

\section{1. Постановка задачи и исходные уравнения}

Пусть: $E$ - модуль упругости, $v$ - коэффициент Пуассона; $c_{1}, c_{2}-$ скорости распространения волн сжатия-растяжения и сдвига; $R_{0}-$ радиус срединной поверхности оболочки, $2 h-$ толщина оболочки: $x, R, \Theta-$ система цилиндрических координат; $t$ - время; $u_{x}, u_{R}-$ компоненты вектора перемещений $\sigma_{x R}, \sigma_{j j}(j=x, R, \Theta)$ - напряжения. Введем следующие безразмерные коэффициенты и координаты:

$$
\begin{gathered}
b_{*}=\frac{R_{0}}{h}, \quad k_{0}^{2}=\frac{c_{2}^{2}}{c_{1}^{2}}=\frac{1-2 v}{2-2 v} \\
\tau=\frac{t c_{2}}{h}, \quad \xi=\frac{x}{h}, \quad \zeta=\xi_{*}-b_{*}, \quad \xi_{*}=\frac{R}{h} .
\end{gathered}
$$

Для конкретности рассмотрим задачу расчета полубесконечной $(x \geqslant 0)$ круговой цилиндрической оболочки при следующих исходных данных:

а) нулевые начальные условия;

б) краевые условия при $x=0$ :

$$
\sigma_{x x}(0, R ; t)=-\frac{C E H(t)}{1-v^{2}}, \quad u_{R}(0, R ; t)=0 ;
$$


в) краевые условия при $R=R_{0} \pm h$ :

$$
\sigma_{x R}\left(x, R_{0} \pm h ; t\right)=\sigma_{R R}\left(x, R_{0} \pm h ; t\right)=0,
$$

где $C=$ const, $H(t)$ - функция Хевисайда.

Будем исследовать следующие безразмерные величины:

$$
\begin{gathered}
u=\frac{u_{x}}{h C}, w=\frac{u_{R}}{h C} \\
\sigma_{11}=\frac{\sigma_{x x}(1+v)}{E C}, \sigma_{13}=\frac{\sigma_{x R}(1+v)}{E C}, \sigma_{33}=\frac{\sigma_{R R}(1+v)}{E C},
\end{gathered}
$$

которые рассмотрим как функции от безразмерных координат $\xi, \zeta, \tau$.

Безразмерные напряжения (1.6) выражаются через безразмерные перемещения (1.5) по формулам

$$
\begin{aligned}
& \sigma_{11}=\frac{1-v}{1-2 v} \frac{\partial u}{\partial \xi}+\frac{v}{1-2 v}\left(\frac{\partial w}{\partial \xi}+\frac{w}{\xi_{*}}\right) \\
& \sigma_{13}=\frac{1}{2}\left(\frac{\partial u}{\partial \xi}+\frac{\partial w}{\partial \xi}\right) \\
& \sigma_{33}=\frac{1-v}{1-2 v} \frac{\partial w}{\partial \zeta}+\frac{v}{1-2 v}\left(\frac{\partial u}{\partial \xi}+\frac{w}{\xi_{*}}\right) .
\end{aligned}
$$

Уравнения равновесия в перемещениях могут быть представлены в форме

$$
\begin{gathered}
\frac{\partial^{2} u}{\partial \zeta^{2}}+\frac{1}{\zeta_{*}} \frac{\partial u}{\partial \zeta}+k_{0}^{-2} \frac{\partial^{2} u}{\partial \xi^{2}}+\frac{1}{1-2 v} \frac{1}{\zeta_{*}} \frac{\partial w}{\partial \xi}+\frac{1}{1-2 v} \frac{\partial^{2} w}{\partial \xi \partial \zeta}=\frac{\partial^{2} u}{\partial \tau^{2}} \\
\frac{\partial^{2} w}{\partial \xi^{2}}+\frac{1}{\zeta_{*}} \frac{\partial w}{\partial \zeta}-\frac{w}{\zeta_{*}^{2}}+k_{0}^{2} \frac{\partial^{2} w}{\partial \xi^{2}}+\frac{1}{2-2 v} \frac{\partial^{2} u}{\partial \xi \partial \zeta}=k_{0}^{2} \frac{\partial^{2} w}{\partial \tau^{2}}
\end{gathered}
$$

Рассмотрим интегрирование системы (1.8). Кроме нулевых началь. ных условий налагаем следующие условия:

1) на основе $(1.6),(1.7)$ из (1.3) вытекают условия

$$
\begin{aligned}
& \text { при } \quad \xi=0: \frac{\partial u}{\partial \xi}=-A, w=0, \\
& \text { где } A=\frac{1-2 v}{(1-v)^{2}}
\end{aligned}
$$

2) на основе $(1.6),(1.7)$ из (1.4) получены условия

$$
\begin{gathered}
\text { при } \zeta= \pm 1: v\left(\frac{\partial u}{\partial \xi}+\frac{w}{ \pm 1+b_{*}}\right)+(1-v) \frac{\partial w}{\partial \xi}=0 \\
\frac{\partial u}{\partial \zeta}+\frac{\partial w}{\partial \xi}=0
\end{gathered}
$$

3) для правильного описания деформации в окрестности первого фронта $\tau=k_{0} \xi$ будем учитывать аналитически выведенные фронтовые условия

$$
\text { при } \tau=k_{0} \xi: u=0, w=0
$$




$$
\frac{\partial u}{\partial \xi}=-A, \frac{\partial w}{\partial \xi}=0
$$

Аналогичным путем нетрудно сформулировать задачу и при других заданных краевых условиях.

\section{2. Расчетный алгоритм}

Пусть $l_{\tau}, l_{\xi}, l_{\xi}$ - шаги прямоугольной сетки по безразмерным координатам $\tau, \xi, \zeta$ соответственно (рис. 1). По соображениям, указанным в [ $\left.{ }^{1}\right]$, выберем следующие соотношения шагов:

$$
l_{\xi}=l_{\zeta}=l, \quad l_{\tau}=\frac{1}{2} k_{0} l_{\xi}
$$

Величину $l$ определим соотношением

$$
l=\frac{1}{J}
$$

где $J$ - целое положительное число.

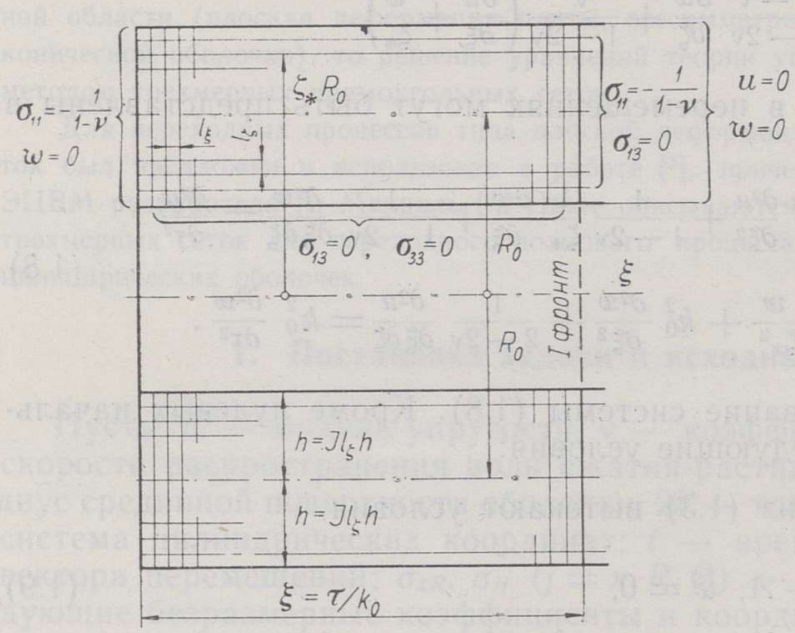

Рис. 1. Продольный разрез оболочки с расчетной сеткой и контурными условиями.

Рассмотрим интегрирование системы (1.8) с очередным выполнением расчета в моменты времени $\tau=n l_{\tau}, \quad(n+1) l_{\tau}$,

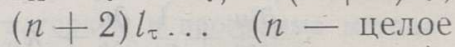
положительное число). Условимся говорить о нечетных слоях, если $n$ нечетное, и о четных слоях, если $n$ четное. На каждом слое (см. рис. 1) будем называть внутренними все точки (узлы сетки), которые не находятся на контуре слоя и в первом ряду $\xi=$ const $3 а$ первым фронтом $\tau=k_{0} \xi$. Последние назовем прифронтовыми точками. В

случае нечетного слоя в прифронтовых точках $\xi=\tau k_{0}^{-1}-\frac{1}{2} l$, а в случае четного слоя $-\xi=\tau k_{0}^{-1}-l$.

Расчет начинается со слоя $\tau=3 l_{\tau}$. На слоях $\tau=3 l_{\tau}, \tau=4 l_{\tau}$ внутренних точек нет.

Начнем с изложения расчетных формул для слоев $\tau=n l_{\tau}, n \geqslant 5$.

1. Во внутренних точках расчет проводится по формулам, полученным из уравнений (1.8) путем замены производных центральными конечно-разностными выражениями первого приближения. Если во внутренней точке $\xi=i l, \xi=j l$ кратко обозначить перемещения через $u_{i j n}$, $w_{i j n}$, то указанные формулы могут быть записаны в следующем виде: 


$$
\begin{aligned}
& u_{i j n}=2 u_{i j, n-1}-u_{i j, n-2}+\frac{1}{4}\left(u_{i+1, j, n-1}-2 u_{i j, n-1}+u_{i-1, j, n-1}\right)+ \\
& +\frac{k_{0}^{2}}{4}\left(u_{i, j+1, n-1}-2 u_{i j, n-1}+u_{i, j-1, n-1}\right)+\frac{k_{0}^{2} l}{8 \zeta_{*}}\left(u_{i, j+1, n-1}-u_{i, j-1, n-1}\right)+ \\
& +\frac{k_{0}^{2}}{16}(1-2 v)^{-1}\left(w_{i+1, j+1, n-1}-w_{i-1, j+1, n-1}-w_{i+1, j-1, n-1}+\right. \\
& \left.+w_{i-1, j-1, n-1}\right)+\frac{k_{0}^{2}}{8}(1-2 v)^{-1} \frac{l}{\zeta_{*}}\left(w_{i+1, j, n-1}-w_{i-1, j, n-1}\right) \\
& w_{i j n}=2 w_{i j, n-1}-w_{i j, n-2}+\frac{k_{0}^{2}}{4}\left(w_{i+1, j, n-1}-2 w_{i j, n-1}+w_{i-1, j, n-1}\right)+ \\
& +\frac{1}{4}\left(w_{i, j+1, n-1}-2 w_{i j, n-1}+w_{i, j-1, n-1}\right)-\frac{l^{2}}{4 \zeta_{*}^{2}} w_{i j, n-1}+ \\
& +\frac{l}{8 \xi_{*}}\left(w_{i, j+1, n-1}-w_{i, j-1, n-1}\right)+\frac{1}{32}(1-v)-1\left(u_{i+1, j+1, n-1}-\right. \\
& \left.\quad-u_{i-1, j+1, n-1}-u_{i+1, j-1, n-1}+u_{i-1, j-1, n-1}\right) .
\end{aligned}
$$

2. На первом фронте $\xi=\tau k_{0}^{-1}$ на основе (1.12) $u=0$, $w=0$.

3. В прифронтовых точках (за исключением точек $\zeta=-1, \zeta=1$ ) $u$ и ш вычисляются из условий (1.13), развернутых при помощи трехточечных односторонних конечно-разностных формул для первых производных по $\xi$.

На нечетных слоях

$$
\begin{aligned}
& u_{\frac{n}{2}-\frac{1}{2}, j n}=\frac{1}{9} u_{\frac{n}{2}-\frac{3}{2}, j n}+\frac{1}{3} A l \\
& w_{\frac{n}{2}-\frac{1}{2}, j n}=\frac{1}{9} w_{\frac{n}{2}-\frac{3}{2}, j n} .
\end{aligned}
$$

На четных слоях

$$
\begin{aligned}
& u_{\frac{n}{2}-1, j n}=\frac{1}{4} u_{\frac{n}{2}-2, j n}+\frac{1}{2} A l \\
& w_{\frac{n}{2}-1, j n}=\frac{1}{4} w_{\frac{n}{2}-2, j n} .
\end{aligned}
$$

4. На краю $\xi=0$ любого слоя условий (1.9), а $u$ определяется для слоя $\tau=n l_{\tau}$ по формуле

$$
u_{0 j n}=\frac{4}{3} u_{1 j n}-\frac{1}{3} u_{2 j n}-\frac{2}{3} A l \text {, }
$$

голученной из первого краевого условия (1.9) путем выражения производной по छ через одностороннюю конечно-разностную формулу.

5. На краях $\zeta= \pm 1$ перемещения $u$, w определяются из системы уравнений, которые строятся следующим образом: 
а) во всех точках, кроме точек $\xi=0, \xi=\tau k_{0}{ }^{-1}$, требуют удовлетворения условий (1.11), $w=0$,

б) в точке $\xi=0$ определяют $u$ по формуле (2.6) и учитывают, что

в) в точке $\xi=\tau k_{0}^{-1}$ используют условия $u=0$,

A. Рассмотрим четный слой $\tau=n l_{\tau}$. Из условия на краю $\zeta=J l=1$ получим следующие уравнения:

$$
\begin{aligned}
& \text { при } i=1 \text { : } \\
& \left.\begin{array}{c}
a u_{1 J n}+w_{2 J n}=A_{11} \\
-\frac{4}{3} u_{1 J n}+b w_{1 J n}+\frac{4}{3} u_{2 J n}=A_{12},
\end{array}\right\} \\
& \text { при } i=\varkappa, 3, \ldots, \frac{n}{2}-1 \text { : } \\
& \left.\begin{array}{l}
-w_{i-1, J n}+a u_{i J n}+w_{i+1, J n}=A_{i 1} \\
-u_{i-1, J n}+b w_{i J n}+u_{i+1, J n}=A_{i 2},
\end{array}\right\}
\end{aligned}
$$

где

$$
\begin{gathered}
A_{12}=\frac{1-v}{v}\left(4 w_{1, J-1, n}-w_{1, J-2, n}\right)+\frac{2}{3} A l \\
A_{i 2}=\frac{1-v}{v}\left(4 w_{i, J-1, n}-w_{i, J-2, n}\right), \quad \text { если } i \geqslant 2 \\
A_{i 1}=4 u_{i, J-1, n}-u_{i, J-2, n}, \quad \text { если } i \geqslant 1 \\
a=3, \quad b=3 \frac{1-v}{v}+\frac{2 l}{1+b_{*}} .
\end{gathered}
$$

Условия (2.7) и (2.8) образуют систему из $n-2$ уравнений. Преобразованием матрицы к верхнетреугольной форме было получено решение в виде следующих рекуррентных формул:

$$
\begin{aligned}
& \left.\begin{array}{c}
u_{i J n}=B_{i 1}-r_{i 1} w_{i+1, J n}-C_{i 1} \\
w_{i J n}=B_{i 2}-r_{i 2} u_{i+1, J n}-C_{t 2}
\end{array}\right\} \quad i<\frac{n}{2}-1 \\
& \left.\begin{array}{l}
u_{i J n}=B_{i 1}-C_{i 1} \\
\Psi_{i J n}=B_{i 2},
\end{array}\right\} \quad i=\frac{n}{2}-1
\end{aligned}
$$

где

$$
\begin{aligned}
& B_{i 1}=r_{i 1}\left(A_{i 1}+B_{i-1,2}\right) \\
& B_{i 2}=r_{i 2}\left(A_{i 2}+B_{i-1,1}+D_{i}\right) \\
& B_{02}=0, \quad B_{12}=\frac{3}{4} r_{12}\left(A_{12}+\frac{4}{3} B_{11}\right)
\end{aligned}
$$

при четных значениях индекса $i$ :

$$
C_{i 1}=S_{i 1} w_{i J n}, \quad C_{i 2}=0, \quad D_{i}=-S_{i-1,1} B_{i-1,2}+S_{i-1,2} B_{i 1},
$$


при нечетных $i$ :

$$
\begin{aligned}
& C_{i 1}=0, \quad C_{i 2}=S_{i 2} w_{i+1, J n}, \quad D_{i}=0 \\
& r_{i 1}=\frac{1}{a+r_{i-1,2}}, \quad r_{i 2}=\frac{1}{b+r_{i-1,1}} \\
& r_{11}=\frac{1}{a}, \quad r_{12}=\frac{4}{3 b} \\
& S_{i 2}=S_{i 1} r_{i 2}, \quad S_{i 1}=S_{i-1,2} r_{i 1}, \quad S_{12}=r_{12} .
\end{aligned}
$$

Условия на краю $\zeta=-J l=-1$ дают систему уравнений, которая отличается от рассмотренной только тем, что

a) $a=-3, \quad b=-3 \frac{1-v}{v}+\frac{2 l}{-1+b_{*}}$,

б) свободные члены $A_{i 1}, A_{i 2}$ имеют противоположные знаки,

в) индексы $J, J-1, J-2$ надо заменить индексами $-J,-(J-1)$, $-(J-2)$.

После таких изменений остаются в силе расчетные формулы $(2.11)-(2.16)$.

Б. На нечетных слоях $\tau=n l_{\tau}$ последний шаг у первого фронта эмеет длину $\frac{1}{2} l$ и расчетные формулы являются следующими:

при $i=1$ сохраняют силу формулы (2.7),

при $i=2,3, \ldots \frac{1}{2}(n-3)$ сохраняют силу формулы $(2.8)$,

при прифронтовых точках $i \doteq \frac{1}{2}(n-1)$ имеют место условия

$$
\left.\begin{array}{l}
-w_{i-1, J n}-\frac{3}{2} a u_{i J n}-3 w_{i J n}=\frac{3}{2} A_{i 1} \\
-u_{i-1, J n}+\frac{3}{2} b w_{i J n}-3 u_{i J n}=\frac{3}{2} A_{i 2} .
\end{array}\right\}
$$

Рекуррентные формулы для решения системы уравнений при $i \leqslant \frac{n-3}{2}$ совпадают с формулами $(2.11)$, а при $\frac{1}{2}(n-1)$ :

$$
\begin{gathered}
u_{i J n}=B_{i 1}+3 r_{i 1} w_{i J n}-C_{i 1} \\
w_{i J n}=r_{i 2}\left(\frac{3}{2} A_{i 2}+B_{i-1,1}+3 B_{i 1}+D_{i}\right) \\
r_{i 1}=\frac{1}{\frac{3}{2} a+r_{i-1,2}} \\
r_{i 2}=\frac{1}{\frac{3}{2} b+r_{i-1,1}+3 r_{i 1}\left[-3+(-1)^{i} S_{i-1,2}\right]},
\end{gathered}
$$

причем на краях $\zeta=+1$ и $\zeta=-1$ величины $a, b$ и свободные члены уравнений имеют различные значения, указанные при рассмотрении случая А.

На первых расчетных слоях $\tau=3 l_{\tau}, \tau=4 l_{\tau}$ существуют только контурные и прифронтовые точки, в которых $и$ и ш вычисляются аналогично вышеизложенному. Разница только в том, что $u_{i j}, u_{i j 4}(i=0,1 ; j=0$, $\pm 1, \pm 2, \ldots, \pm J)$ приходится вычислять из систем уравнений. Значения $\bar{u}$ и

3 ENSV TA Toimetised F-1 66. 
Таблища

\begin{tabular}{c|c|c|c|c|c}
\hline \multirow{2}{*}{$j$} & \multirow{2}{*}{$i$} & \multicolumn{2}{|c|}{ Слой $\tau=3 l_{\tau}$} & \multicolumn{2}{c}{ Слой $\tau=4 l_{\tau}$} \\
\cline { 3 - 6 } & & $u_{i j 3}$ & $w_{i j 3}$ & $u_{i j 4}$ & $w_{i j 4}$ \\
\hline$-J<j<J$ & 0 & 0,1224489 & 0 & 0,1632653 & 0 \\
& 1 & 0,04081632 & 0 & 0,08163265 & 0 \\
\hline$j=-J$ & 0 & 0,1628910 & 0 & 0,1632653 & 0 \\
& 1 & 0,0632840 & $-0,0337015$ & 0,08163265 & $-0,0233979$ \\
\hline$j=J$ & 0 & 0,1625563 & 0 & 0,1632653 & 0 \\
& 1 & 0,06309819 & 0,0334228 & 0,08163265 & 0,0232632
\end{tabular}

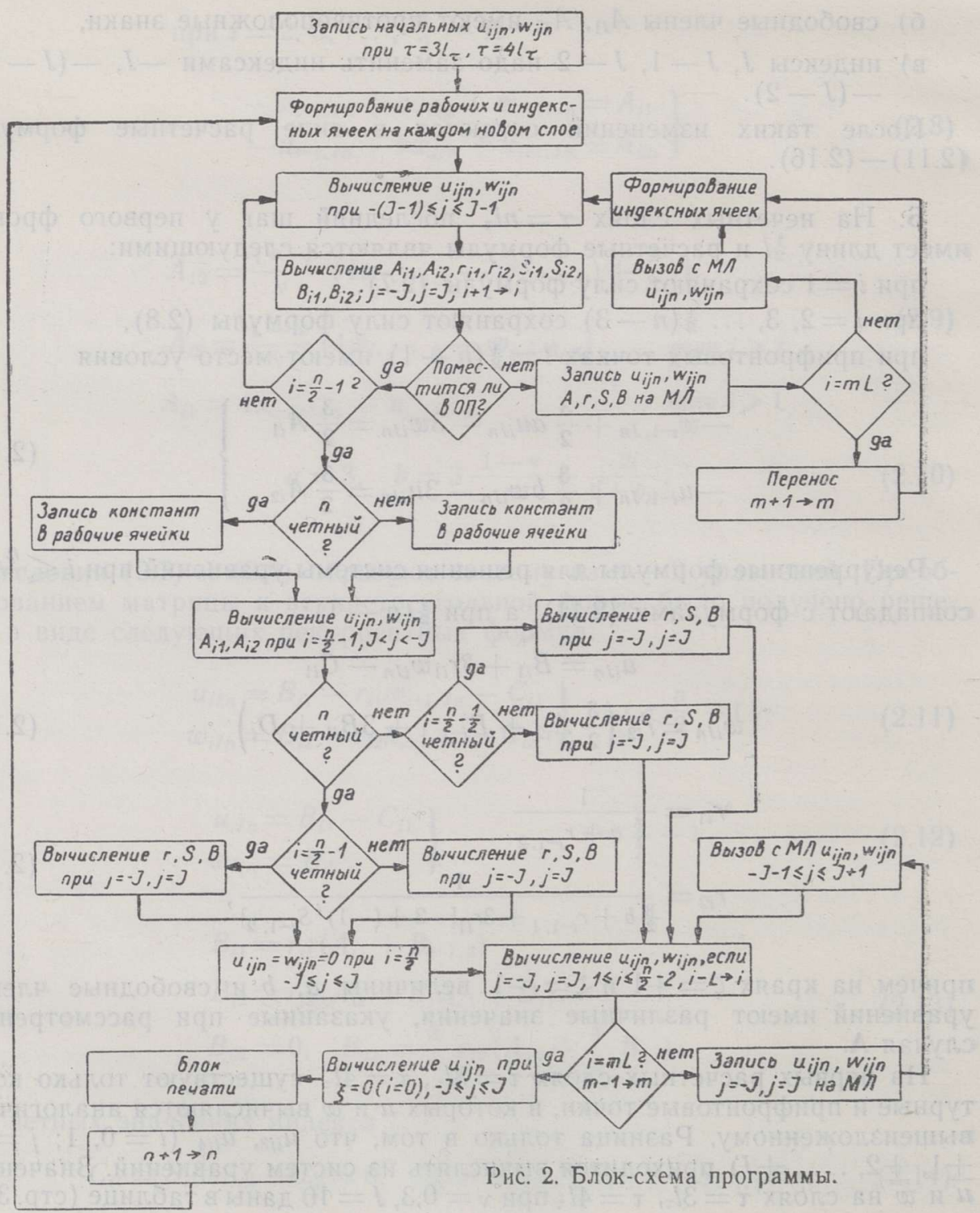




\section{3. Блок-схема программы}

Расчеты были реализованы при помощи ЭЦВМ «Минск-2», оперативная память (ОП) которой составляет 4096 слов. Указанный объем ОП позволяет провести расчет до слоя $\tau=72 l_{\tau}$ включительно. Для продолжения расчета была использована внешняя память (магнитные ленты - МЛ). Блок-схема программы показана на рис. 2.

Последовательность расчета:

а. Вычисления во внутренних точках сетки. На каждом слое $\tau=n l_{\tau}$ сначала вычисляются $u_{i j n}, w_{i j n}$ во всех внутренних точках $j=0$, \pm 1 , $\pm 2, \pm 3, \ldots, \pm(J-1), i=1,2,3, \ldots, N$, где $N=\frac{n}{2}-2$ на четных слоях и $N=\frac{n}{2}-\frac{3}{2}$ на нечетных слоях. При каждом фиксированном $i$ одновременно вычисляются также вспомогательные величины $A_{i 1}, A_{i 2}$, $r_{i 1}, r_{i 2}, S_{i 1}, S_{i 2}$. При этом первая группа рядов, вычисляемых без обмена информацией между ОП и $M Л$, содержит $L$ рядов, а каждая следующая группа - 3 вычисленных ряда и еще до $L-3$ новых рядов.

б. Вычисление $u_{i j n}, w_{i j n}$ в прифронтовом ряде при $j=0, \pm 1$, $\pm 2, \ldots, \pm J$

в. Вычисление $u_{i j n}, w_{i j n}$ при $j= \pm J$.

д. Вычисление $u_{0 j n}$ при $j=0, \pm 1, \pm 2, \ldots, \pm J$.

Объем программы - примерно $1200(10)$ слов.

В конкретных расчетах были приняты следуюшие численные коэффициенты:

$$
b_{*}=10, v=0,30, J=10, \max n=800 .
$$

Продолжительность расчета - примерно 60 часов.

\section{ЛИТЕРА Т У Р А}

1. А йн о л а Л., Н иг у л У., Изв. АН ЭССР. Сер. физ.-матем. и техн. наук, 14, № 1 , 3-63 (1965).

2. Н иг ул У., Изв. АН ЭССР. Сер. физ.-матем. и техн. наук, 14, № 3, 345-384 (1965).

3. М янн и ль А., Изв. АН ЭССР. Сер. физ.-матем. и техн, наук, 14, № $3,423-427$ (1965).
Институт кибернетики
Академии наук Эстонской ССР
Поступила в редакцию
$15 / \mathrm{X} \quad 1965$

\section{U. NIGUL, M. PETERSON}

\section{KOLMEMÕ̃TMELISE VÕRGUMEETODI ALGORITM DUNAAMILISTE ULEMINEKUPROTSESSIDE UURIMISEKS SILINDRILISES KOORIKUS TELGSUMMEETRILISE DEFORMATSIOONI KORRAL}

Järsu koormamise korral koorikuis esilekutsutud pingelaineid on seni võrdlemisi vähe uuritud. Olemasolevad tööd (vt. [1]) baseeruvad ligikaudseil koorikute teooriail.

Käesolevas artiklis esitatakse algoritm ja programm elastsusteooria võrrandite numbriliseks integreerimiseks kolmemõötmelise võrgumeetodiga silindrilise kooriku puhul. Kirjeldatav algoritm on töös [ $\left.{ }^{2}\right]$ plaadi jaoks koostatud algoritmi üldistuseks. Tal on järgmised olulised eriomadused: a) kasutades valemeid (1.12) ja (1.13), arvestatakse pingete katkevust esimesel frondil; b) kooriku pindadel $R=R_{0} \pm h$ pingete nulliga võrdumise tingimustest (1.11) tuletatud võrrandisüsteemi lahendid antakse rekurrentsete valemitega (2.7) $-(2.19)$.

Programmeerimisküsimusi vaadeldakse arvuti «Minsk-2» võimalusi arvestades. 
U. NIGUL, M. PETERSON

\section{ALGORITHM OF THE THREE-DIMENSIONAL NETWORKS METHOD FOR AN ANALYSIS OF AXISYMMETRIC TRANSIENT STRESS WAVES IN A CYLINDRICAL SHELL}

Up to the present time, only a small number of investigations [1] have been devoted to transient stress waves generated in shells by the application of impulsive dynamic loads. All these investigations have been carried out by using approximate shell theories.

This paper offers an algorithm and program for a numerical integration of equations of the theory of elasticity for a circular cylindrical shell by a method of finite differences in which a fixed three-dimensional rectangular mesh is used. The algorithm described here is a generalization of that proposed in $\left.{ }^{2}\right]$ for plates. Its most important features are as follows: a) the stress discontinuity on the first front is taken into consideration by using $(1.12),(1.13)$; b) the solutions of the systems of equations derived from the "zero-stress-conditions" (1.11) at $R=R_{0} \pm h$ are given in the form of recurrent formulae $(2.7)-(2.19)$.

The programming problems are discussed, taking into consideration the possibilities of the computer "Minsk-2" used by the authors in practical computations. 